\title{
Exploring current physicians' failure to communicate clinical feedback back to transferring physicians after transitions of patient care responsibility: A mixed methods study
}

\author{
Judith L. Bowen · Joseph Chiovaro · Bridget C. O’Brien · Christy Kim Boscardin · David M. Irby · Olle ten Cate
}

Published online: 8 June 2020

(c) The Author(s) 2020

\begin{abstract}
Introduction After patient care transitions occur, communication from the current physician back to the transferring physician may be an important source of clinical feedback for learning from outcomes of previous reasoning processes. Factors associated with this communication are not well understood. This study clarifies how often, and for what reasons, cur-
\end{abstract}

This research was conducted at Oregon Health and Science University and the Portland Veterans Affairs Health

Care System. The work herein should be attributed to: Department of Medicine, Oregon Health and Science University, Portland, Oregon, USA.

Electronic supplementary material The online version of this article (https://doi.org/10.1007/s40037-020-00585-1) contains supplementary material, which is available to authorized users.

\section{J. L. Bowen (ه)}

Department of Medical Education and Clinical Sciences, Elson S Floyd College of Medicine, Washington State University, Spokane, WA, USA

judith.bowen@wsu.edu

\section{J. Chiovaro}

Department of Medicine, Division of Hospital Medicine,

Oregon Health and Science University, and Portland

Veterans Affairs Healthcare System, Portland, OR, USA

\section{B. C. O'Brien · C. K. Boscardin · D. M. Irby}

Department of Medicine and Center for Faculty Educators,

University of California San Francisco School of Medicine,

San Francisco, CA, USA

\section{O. ten Cate}

Center for Research and Development of Education, University Medical Center Utrecht, Utrecht University, Utrecht, The Netherlands

Center for Faculty Educators, University of California San Francisco School of Medicine, San Francisco, CA, USA rent physicians do or do not communicate back to transferring physicians about transitioned patients. Methods In 2018, 38 physicians at two academic teaching hospitals were interviewed about communication decisions regarding 618 transitioned patients. Researchers recorded quantitative and qualitative data in field notes, then coded communication rationales using directed content analysis. Descriptive statistics and mixed effects logistic regression analyses identified communication patterns and examined associations with communication for three conditions: When current physicians 1) changed transferring physicians' clinical decisions, 2) perceived transferring physicians' clinical uncertainty, and 3) perceived transferring physicians' request for communication. Results Communication occurred regarding $17 \%$ of transitioned patients. Transferring physicians initiated communication in $55 \%$ of these cases. Communication did not occur when current physicians 1) changed transferring physicians' clinical decisions (119 patients), 2) perceived transferring physicians' uncertainty (97 patients), and 3) perceived transferring physicians' request for communication (12 patients). Rationales for no communication included case contextual, structural, interpersonal, and cultural factors. Perceived uncertainty and request for communication were positively associated with communication $(p<0.001)$ while a changed clinical decision was not.

Discussion Current physicians communicate infrequently with transferring physicians after assuming patient care responsibilities. Structural and interpersonal barriers to communication may be amenable to change. Clarity about transferring physicians' uncertainty and desire for communication back may improve clinical feedback communication. 
Keywords Clinical feedback · Patient care transitions · Communication · Clinical reasoning processes

\section{Introduction}

Transitions of responsibility for patient care are common in modern healthcare settings. As a consequence of several forces, including efficiency demands, patient safety, and specialization, physicians often work as site-based specialists in designated blocks of time [1-3]. These structures require transferring physicians, those initially responsible for clinical reasoning decisions, to transition patients to current physicians. Unlike temporary patient handovers where physicians subsequently return to care for the same patients, these transitions disrupt the transferring physician's clinical reasoning processes. Outcomes of decisions they made before the transition may not become easily known unless follow-up occurs. These outcomes of prior decisions are a form of clinical feedback-information embedded in the clinical reasoning process as the patient's condition evolves-that help physicians calibrate their future clinical decisions and learn [4-7]. While efforts to close this clinical feedback loop have been described, physicians may not seek outcome information about all their patients $[6,8,9]$. After-transition communication from current physicians back to transferring physicians could also close this clinical feedback loop, but the frequency of and factors associated with this communication are not well understood. If communication does not occur, opportunities to learn and improve future clinical reasoning processes may be lost [5].

Researchers have documented verbal[10] and nonverbal[11] communication challenges during patient care transitions and made recommendations for studying[12] and improving the quality of these transitions to address patient safety concerns [11, 13-17]. While communication about clinical outcomes may take place post-hoc for a small number of patients with unfortunate outcomes in the form of morbidity and mortality conferences[18], no studies address communication from current physicians back to transferring physicians after care transitions have occurred and when patients' clinical trajectories have evolved. Factors associated with communicating clinical feedback about transitioned patients as part of physicians' daily work have not been identified.

When viewed through the lens of situated cognition theory, interactions between individuals and their work environments are integral to learning from work performance such as caring for patients [19, 20]. Situated cognition posits that knowing and doing are inseparable because knowledge is embedded in the social, cultural, and physical contexts in which it occurs. From this perspective, learning occurs during activities in which individuals and context interact in ways that produce new knowledge, meaning, or in- sight. Thus, the activity of caring for patients with particular problems in clinical contexts with particular cultures, relationship expectations, and resources will shape learning [19]. In contexts where transitions of patient care responsibility occur, discontinuity is an important contextual dimension. Three conditions specific to the dimension of discontinuity may influence transferring physicians' learning via a current physician's decision to communicate back about a patient's outcome. First, when the care transition occurs, current physicians may perceive transferring physicians to express uncertainty about diagnoses or management decisions for complex clinical cases and wish to assist with reducing that uncertainty via clinical feedback [21, 22]. Second, if transferring physicians express a desire to know what happened and request communication back, the current physician may then be more likely to share clinical feedback. Third, when current physicians change transferring physicians' clinical decisions, anticipation that transferring physicians will be receptive to clinical feedback in these situations may be important [21, 23-25]. Thus, the very structures that require transitions of responsibility may also influence whether and how communication occurs, and what transferring physicians' learn when transitions disrupt their clinical reasoning processes.

In order to better understand how to support transferring physicians' learning in the context of discontinuity, we must first understand why current physicians do or do not communicate back to transferring physicians. Therefore, the purpose of this study is to clarify[26], in the context of actual practice, in what situations and for what reasons current physicians do or do not communicate with transferring physicians about transitioned patients for whom transferring physicians are no longer responsible.

\section{Methods}

\section{Study design}

We employed a convergent, mixed methods design, collecting both quantitative and qualitative data at the same time to develop a more comprehensive understanding of current physicians' communication patterns and their rationales in the context of patient care transitions [27]. We used the qualitative data to amplify the quantitative data. We defined patient care transition as a formal transition of responsibility for patients that occurs when the transferring physician will no longer have any ongoing responsibility. We distinguish these formal transitions from handovers that occur at the end of shifts when covering physicians accept temporary responsibility and the primary physician returns the next day to resume responsibility for the same patients.

Several research questions guided our inquiry. For the quantitative strand of this study, we asked: 1) How 
Table 1 Conditions of interest (predictor variables) and the outcome variable, and associated structured interview questions for quantitative and qualitative strands

\begin{tabular}{|c|c|c|c|}
\hline & \multirow[b]{2}{*}{ Definition } & Quantitative data & \multirow[t]{2}{*}{ Qualitative data } \\
\hline & & Structured interview questions & \\
\hline \multicolumn{4}{|l|}{$\begin{array}{l}\text { Condition of interest (Pre- } \\
\text { dictor variable) }\end{array}$} \\
\hline $\begin{array}{l}\text { Perceived transferring } \\
\text { physicians' uncertainty }\end{array}$ & $\begin{array}{l}\text { The current physician perceived the } \\
\text { transferring physician to express un- } \\
\text { certainty about clinical decisions at the } \\
\text { time of the care transition }\end{array}$ & $\begin{array}{l}\text { Did the transferring hospitalist express } \\
\text { uncertainty about the diagnosis or } \\
\text { management for this patient at the } \\
\text { time of transition? (yes/no) }\end{array}$ & \\
\hline $\begin{array}{l}\text { Perceived transferring } \\
\text { physicians' request for } \\
\text { communication }\end{array}$ & $\begin{array}{l}\text { The current physician perceived the } \\
\text { transferring physician to request com- } \\
\text { munication back about the case at the } \\
\text { time of the care transition }\end{array}$ & $\begin{array}{l}\text { Did the transferring hospitalist request } \\
\text { communication back about this pa- } \\
\text { tient? (yes/no) }\end{array}$ & \\
\hline Changed clinical decision & $\begin{array}{l}\text { The current physician changed the } \\
\text { transferring physicians' diagnosis } \\
\text { or management plan after the care } \\
\text { transition }\end{array}$ & $\begin{array}{l}\text { Did you change the patient's diagnosis } \\
\text { or management plan after the transi- } \\
\text { tion? (yes/no) }\end{array}$ & \\
\hline \multicolumn{4}{|l|}{ Outcome variable } \\
\hline Communication occurred & $\begin{array}{l}\text { The current physician reported the } \\
\text { status of communication with the trans- } \\
\text { ferring physician }\end{array}$ & $\begin{array}{l}\text { Did you communicate back about this } \\
\text { patient? (yes/no) }\end{array}$ & $\begin{array}{l}\text { If yes, what was the primary reason for the } \\
\text { communication? } \\
\text { Who initiated the communication? } \\
\text { What was the method of communication? } \\
\text { If no, what was the primary reason commu- } \\
\text { nication did not occur? }\end{array}$ \\
\hline
\end{tabular}

often do current physicians communicate with transferring physicians about patients that transitioned between them? And, 2) What are the associations between communication and the conditions we hypothesized might influence whether or not current physicians communicate back to transferring physicians? We selected communication occurrence about a patient as the primary outcome variable. To explore associations with a communication occurrence, we selected three situational conditions of interest amenable to quantitative responses as shown in Tab. 1: a) when current physicians perceived transferring physicians' uncertainty, b) when current physicians perceived transferring physicians' request for communication, and c) when current physicians changed transferring physicians' clinical decisions. For the qualitative strand, we asked: how do current physicians describe their rationales for communicating or not communicating back to transferring physicians (Tab. 1)? The institutional review boards at Oregon Health \& Science University and the Portland Veterans Affairs Health Care System approved the study (IRB ID: STUDY00015374, May 16, 2018). The principal investigator (JB) obtained informed consent from all study participants. This work was carried out in accordance with the Declaration of Helsinki, including but not limited to, there being no potential harm to participants, that the anonymity of participants was guaranteed, and that informed consent was obtained from each participant.

\section{Setting}

This study took place at two academic teaching hospitals. Participants were members of academic hospital- ist group practices at one or the other hospital. Physicians in these work groups are variably assigned to the traditional teaching service or the clinical hospitalist service. The teaching service physician supervises patient care provided by teams of learners, including resident physicians, medical students, and physician assistant students. The clinical hospitalist service physician provides direct care for patients with only rare involvement with trainees. Work assignments for physicians are 1-2 weeks in duration. The transitions of interest occurred when the transferring physician transitioned patients to the current physician at the end of these 7 - or 14-day work assignments.

\section{Participants}

We recruited internal medicine physicians with established working relationships with members of their practice group who routinely transition patient care responsibility between each other. We invited physicians to participate via weekly email messages until no further responses were received for three consecutive weeks. Following consent, work assignments were used to contact current physicians to schedule interviews approximately 4 days after the participant (current physician) had received patient care responsibility from the transferring physician. We chose to collect data 4 days after the patient care transition based on the average length-of-stay in both hospitals, working under the assumption that most of the diagnostic and dispositional decisions would have occurred by then and could serve as potential sources of clinical feedback communication. 


\section{Data collection}

We collected data during brief structured interviews (on average, one minute per transitioned patient); closed-ended questions yielded categorical data for the quantitative analysis, open-ended questions yielded descriptive data for the qualitative analysis (Tab. 1). The principal investigator, JB, conducted all of the interviews while participants were still on service and responsible for patient care. Because work assignments varied, JB conducted one to six interviews per participant. Using their structured signout sheet to refer anonymously to patients received in transition, JB asked participants questions about each transitioned patient and recorded quantitative responses and field notes using data collection forms to assure consistent data collection (Tab. 1). When possible, field notes captured verbatim phrases and were recorded as such; these are reported with quotations marks. JB transcribed field notes after each interview and entered anonymized categorical and descriptive data about each transitioned patient into a spreadsheet.

\section{Analysis}

Quantitative analysis To assess the study's effect on communication for participants interviewed more than once, we first calculated the communication frequency by interview number to monitor for changes in communication behavior over time. We found no increase in feedback communication during the study period [28]; therefore, we included all of the data in the analyses. We used two approaches to explore associations between communication occurrence and the three conditions of interest: 1) perceived uncertainty, 2) perceived communication request, and 3) changed clinical decisions. First, we used descriptive statistics to determine the overall communication rate and communication frequencies for each condition. Next, we examined the effect of each condition on the binary outcome, communication versus no communication. To account for the nested structure of the data, we conducted bi-variable mixed effects logistic regression analyses using each condition as a predictor variable (Stata SE 13.1 for Windows, Stata Corp, College Station, Texas). Then, all three predictors were included in the final multivariable mixed-effects logistic regression model to determine the relative contributions of each as predictors on communication outcome.

Qualitative analysis Two researchers (JB and JC) independently analyzed all participants' anonymized rationales for communication decisions from the interviews. JB is a physician and education researcher with previous experience as a physician on a traditional teaching service at one of the study sites but has no current work relationship with participants at either site. Her experience allowed for shared understanding of the colloquial terms participants used during the interviews. JC is an academic physician with current experience on both teaching and clinical hospitalist services at the other study site. This researcher's insights about workflow facilitated timing and location of interviews. To address his potentially compromising role, he did not serve as a study subject nor conduct any interviews. Hospital identity was removed prior to JC's involvement in the analysis. JB and JC developed an initial coding structure based on factors previously found to influence communication between current and transferring physicians [21]. Following directed qualitative content analytic procedures [29], we applied the following codes for the rationales provided: (1) case contextual (e.g. specific case details), (2) structural (e.g. work proximity), and (3) interpersonal (hierarchy, familiarity, relationship). Rationales not fitting these definitions were coded as (4) other. After independently coding a subset of rationales, we discussed findings, revised the codebook, and both researchers independently applied the codes to the entire data set derived from interview field notes. We negotiated differences through discussion. A third researcher, $\mathrm{BO}$, an experienced medical education qualitative researcher, provided oversight to check interpretations throughout the analytic process. The final codebook is available as Supplemental Digital Appendix 1. We calculated occurrence rates for coded rationales to display frequencies [30]. In $2 \%$ of cases, we identified more than one rationale but included only the first for frequency calculations.

\section{Results}

The principal investigator (JB) interviewed 21 Site 1 physicians (78\% of eligible hospitalists) and 17 Site 2 physicians (71\% of eligible hospitalists) over 3 months (May through July) in 2018. Twenty (53\%) self-identified as male and $18(47 \%)$ as female. JB conducted 94 interviews, 41 at Site 1 and 53 at Site 2. Each participant contributed an average of 2.5 interviews and discussed an average of 6.6 patients per interview. Participants transitioned 618 patient cases. As shown in Tab. 2, participants reported similar experiences by site, although current physicians changed transferring physicians' clinical decisions more often at Site 1 .

Next, we report results for our primary outcome, when communication occurred, and associations of communication with the conditions of interest: a) perceived uncertainty, b) perceived communication request, and c) changed clinical decisions (Tab. 1.) We use qualitative results to elaborate why communication occurred. Then we report results when communication did not occur under the same conditions and use qualitative results to elaborate why communication did not occur. 
Table 2 Study site comparisons for numbers and frequency of communication occurrences and conditions of interest for all 618 patients transitioned during the study period in two academic hospitalist practices in 2018

\begin{tabular}{|c|c|c|c|}
\hline & $\begin{array}{l}\text { Site } 1 \\
N, \%\end{array}$ & $\begin{array}{l}\text { Site } 2 \\
N, \%\end{array}$ & $\begin{array}{l}\text { Total } \\
N, \%\end{array}$ \\
\hline Patients transitioned during study period & $260,42 \%$ & $358,58 \%$ & $618,100 \%$ \\
\hline Communication occurrences & $39,15 \%$ & $68,19 \%$ & $107,17 \%$ \\
\hline \multicolumn{4}{|l|}{ Frequency of condition of interest } \\
\hline Current physician perceived transferring physician's uncertainty & $69,26 \%$ & $84,23 \%$ & $153,25 \%$ \\
\hline Current physician perceived transferring physician's communication request & $23,9 \%$ & $25,7 \%$ & $48,8 \%$ \\
\hline Current physician changed transferring physician's clinical decision(s) & $95,36 \%$ & $74,21 \%$ & $169,27 \%$ \\
\hline
\end{tabular}

\section{When communication occurred}

Communication between current and transferring physicians occurred in 107 of 618 (17\%) cases. Of these, the transferring physician initiated communication $55 \%$ of the time, and these communications occurred face-to-face $58 \%$ of the time. As shown in Tab. 3, communication was positively associated with all three conditions in the mixed effects bivariable logistic regression analysis. However, in the final mixed effects multivariable logistic regression model, current physicians' perceptions of transferring physicians' uncertainty and request for communication were positively and significantly associated with communication occurrence but changing transferring physicians' clinical decisions was not significantly associated with communication.

Participants' (current physicians') rationales for communicating back under the three conditions of interest appear in Tab. 4. Rationales for most communication occurrences across all conditions involved case contextual factors. For example, current physicians provided clinical updates intended to "clarify," "inform," and "confirm." Some rationales included elaborate descriptions, such as sharing reasons for why the patient was transferred to the intensive care unit for more aggressive treatment of refractory heart failure. In a few examples, interpersonal factors facilitated communication, such as knowing the transferring physician was "clinically curious". Occasionally, participants used the word "collaborated" to describe working together on subsequent clinical decisions.

\section{When communication did not occur}

Of cases where communication did not occur, the most common situation was that in which the transferring physician communicated confidence in the clinical assessment and the current physician subse- quently agreed, made no changes to the transferring physician's clinical decisions, and the clinical course turned out "as expected" ( $n=362$ out of $511,71 \%$ ). We explored further the 228 cases and their rationales where communication did not occur even though transferring physicians were perceived to have expressed uncertainty, and/or requested communication, and/or current physicians changed transferring physicians' clinical decisions (Tab. 5).

As shown in Tab. 5, although we might have expected perceived uncertainty to serve as an invitation to communicate with transferring physicians, communication did not occur for most of these cases (97 of 156, Tab. 5, first condition.) When the transferring physician was perceived to invite communication, communication did not occur for some of these cases (12 of 50, Tab. 5, second condition). Current physicians changed the transferring physicians' clinical decisions in 169 cases and communication did not occur in 119 of them (Tab. 5, third condition).

\section{Rationales for no communication across three conditions}

Case contextual factors were frequent explanations in all cases of no communication. When providing these rationales, participants occasionally appeared to make judgments about the lack of learning value to the transferring physician (i.e. clinically uninteresting). We also noticed the rationale, "course as expected", suggesting the current physician's perception that the patient's evolving clinical picture was consistent with the transferring physician's anticipatory guidance, even though the current physician perceived uncertainty on the part of the transferring physician. We found rationales related to structural problems interfering with communication, including unavailable information at the time of the interview and lack of opportunity due to work schedules and

Table 3 Mixed effects logistic regression analysis examining the conditions associated with communication occurrence

\begin{tabular}{|lcc|ccc|c|} 
& \multicolumn{1}{c}{ Mixed effects bi-variable logistic regression } & \multicolumn{4}{c|}{ Mixed effects multivariable logistic regression } \\
& OR & $95 \% \mathrm{Cl}$ & $P$-value & OR & $95 \% \mathrm{Cl}$ & $P$-value \\
\hline Perceived uncertainty & 6.1 & $3.7,10.4$ & $<0.001$ & 3.0 & $1.6,5.8$ & $<0.001$ \\
\hline Perceived communication request & 41.4 & $15.8,108.3$ & $<0.001$ & 22.2 & $8.3,59.0$ & $<0.001$ \\
\hline Changed clinical decision & 3.7 & $2.3,6.3$ & $<0.001$ & 1.4 & $0.7,2.7$ & 0.290
\end{tabular}


Table 4 Current physicians' communication frequency and rationales for communicating back to transferring physicians for 107 out of 618 cases where communication occurred in two academic hospitalist practices in 2018

\begin{tabular}{|c|c|c|c|c|c|c|c|}
\hline & & \multicolumn{6}{|c|}{ Condition of interest } \\
\hline & & \multicolumn{2}{|c|}{$\begin{array}{l}\text { Perceived transferring physicians' uncer- } \\
\text { tainty }\end{array}$} & \multicolumn{2}{|c|}{$\begin{array}{l}\text { Perceived transferring physicians' com- } \\
\text { munication request }\end{array}$} & \multicolumn{2}{|c|}{$\begin{array}{l}\text { Changed transferring physicians' clinical } \\
\text { decisions }\end{array}$} \\
\hline & & Yes & No & Yes & No & Yes & No \\
\hline \multirow{2}{*}{$\begin{array}{l}\text { Communication } \\
\text { occurred }\end{array}$} & Yes & $59,9 \%$ & $48,8 \%$ & $38,6 \%$ & $69,11 \%$ & $50,8 \%$ & $57,9 \%$ \\
\hline & No & $97,16 \%$ & $414,67 \%$ & $12,2 \%$ & $499,81 \%$ & $119,19 \%$ & $392,64 \%$ \\
\hline \multicolumn{2}{|c|}{$\begin{array}{l}\text { Frequency of rationales } \\
\text { by category for commu- } \\
\text { nication }\end{array}$} & \multicolumn{2}{|c|}{$\begin{array}{l}\text { For all } 59 \text { communication occurrences } \\
\text { when transferring physician expressed } \\
\text { uncertainty }(n, \%)\end{array}$} & \multicolumn{2}{|c|}{$\begin{array}{l}\text { For all } 38 \text { communication occurrences } \\
\text { when transferring physician requested } \\
\text { communication }(n, \%)\end{array}$} & \multicolumn{2}{|c|}{$\begin{array}{l}\text { For all } 50 \text { communication occurrences } \\
\text { when clinical decisions changed ( } n, \%)\end{array}$} \\
\hline \multicolumn{2}{|c|}{ Case contextual factors } & \multicolumn{2}{|l|}{$46,78 \%$} & \multicolumn{2}{|l|}{$33,87 \%$} & \multicolumn{2}{|l|}{$38,76 \%$} \\
\hline \multicolumn{2}{|c|}{ Structural factors } & \multicolumn{2}{|l|}{$2,3 \%$} & \multicolumn{2}{|l|}{$0,0 \%$} & \multicolumn{2}{|l|}{$3,6 \%$} \\
\hline \multicolumn{2}{|c|}{ Interpersonal factors } & \multicolumn{2}{|l|}{$8,14 \%$} & \multicolumn{2}{|l|}{$4,10 \%$} & \multicolumn{2}{|l|}{$7,14 \%$} \\
\hline \multicolumn{2}{|c|}{ Other } & \multicolumn{2}{|l|}{$3,5 \%$} & \multicolumn{2}{|l|}{$1,3 \%$} & \multicolumn{2}{|l|}{$2,4 \%$} \\
\hline \multicolumn{2}{|l|}{ Category } & \multicolumn{6}{|c|}{ Examples of rationales for communication across 3 conditions } \\
\hline \multicolumn{2}{|c|}{ Case contextual factors ${ }^{\mathrm{a}}$} & \multicolumn{6}{|c|}{$\begin{array}{l}\text { - Provided update on clinical course } \\
\text { - Clarified how current physician figured out complex medication refill history } \\
\text { - Discussed challenging management situation } \\
\text { - Updated and educated on rare diagnosis } \\
\text { - Obtained more information about big picture to execute discharge } \\
\text { - Explained reason for stopping antibiotics }\end{array}$} \\
\hline \multicolumn{2}{|c|}{ Structural factors ${ }^{b}$} & \multicolumn{6}{|c|}{$\begin{array}{l}\text { - Ran into each other } \\
\text { - Secondary to discussion of other patients }\end{array}$} \\
\hline \multicolumn{2}{|c|}{ Interpersonal factors ${ }^{c}$} & \multicolumn{6}{|c|}{$\begin{array}{l}\text { - Discussed and co-managed, allowed nuanced tailoring of treatment } \\
\text { - Provided reassurance that nothing was missed } \\
\text { - Based on prior working relationship, knew she would want to know } \\
\text { - Transferring physician wondered about current physician's perception of his decision-making; conversation supportive, debriefed } \\
\text { patient's death }\end{array}$} \\
\hline \multicolumn{2}{|l|}{ Other ${ }^{d}$} & \multicolumn{6}{|c|}{$\begin{array}{l}\text { - Kept everyone in the loop } \\
\text { - Provided clinical update in front of physician assistants; pointed out work up recommended but not carried out } \\
\text { - Communicated "thanks" back to transferring physician at family's request }\end{array}$} \\
\hline \multicolumn{8}{|c|}{$\begin{array}{l}\text { aRationales pertaining to the clinical case, including judgments about the learning value for the transferring physician } \\
\text { b Rationales pertaining to structural barriers including clinical information not yet available, no opportunity to communicate (i.e. on vacation), time constraints, or } \\
\text { communication is scheduled in the future because the current physician will be transitioning patients back to the same physician } \\
\text { CRationales pertaining to interpersonal factors including barriers related to hierarchy, familiarity with how the transferring physician will react, or no established } \\
\text { relationship } \\
\text { d Rationales that did not fit into the above categories including references to the culture of communication or hedging efforts to communicate. }\end{array}$} \\
\hline
\end{tabular}

vacations. Participants described barriers of hierarchy ("it would be uncomfortable [for me] to tell my boss he was wrong") and challenging relationships with transferring physicians ("felt uneasy about our relationship"). Familiarity with transferring physicians' reactions to clinical feedback, when negative (i.e. "defensive posture"), appeared to inhibit efforts to communicate. Knowing transferring physicians' habits of "chart stalking" to find needed information led some to assume communication was not needed. Finally, we observed rationales we attributed to the workplace culture (coded as other). For example, participants described communicating back as not "how we operate" in their group practice.

\section{Discussion}

Researchers have studied communication challenges at the time of patient care transitions [11-15]. We explored transition communication from a different perspective-from current to transferring physicians to provide follow-up after transitions of responsibil- ity had taken place. We observed a low communication frequency-only $17 \%$-among physicians working in established hospitalist group practices. Conducting our study of discontinuity from the perspective of situated cognition illuminated several conditions that influence current physicians' communication back to transferring physicians and may therefore impact learning and refinement of clinical reasoning. When communication did occur, it was associated with current physicians' perceptions of transferring physicians' clinical uncertainty and their requests for communication feedback. When communication did not occur, some conditions functioned as communication deterrents from the perspective of the current physician. Cases that turned out as expected were perceived to have low learning value. Structures such as opposite work schedules and competing patient care priorities deterred communication. Current physicians avoided communicating when relationship factors such as hierarchy and previous communication experiences were challenging. Lack of a commu- 
Table 5 Current physicians' frequencies and rationales for no communication back to transferring physicians for 228 out of 618 transitioned patients in two academic physician practices in 2018

\begin{tabular}{|c|c|c|c|c|c|c|c|}
\hline & & \multicolumn{6}{|c|}{ Condition of interest } \\
\hline & & \multicolumn{2}{|c|}{$\begin{array}{l}\text { Perceived transferring physicians' uncer- } \\
\text { tainty }\end{array}$} & \multicolumn{2}{|c|}{$\begin{array}{l}\text { Perceived transferring physicians' commu- } \\
\text { nication request }\end{array}$} & \multicolumn{2}{|c|}{$\begin{array}{l}\text { Changed transferring physicians' clinical } \\
\text { decisions }\end{array}$} \\
\hline & & Yes & No & Yes & No & Yes & No \\
\hline \multirow{2}{*}{$\begin{array}{l}\text { Communication } \\
\text { occurred }\end{array}$} & Yes & $59,9 \%$ & $48,8 \%$ & $38,6 \%$ & $69,11 \%$ & $50,8 \%$ & $57,9 \%$ \\
\hline & No & $97,16 \%$ & $414,67 \%$ & $12,2 \%$ & $499,81 \%$ & $119,19 \%$ & $392,64 \%$ \\
\hline \multicolumn{2}{|c|}{$\begin{array}{l}\text { Frequency of rationales } \\
\text { by category for no } \\
\text { communication }\end{array}$} & \multicolumn{2}{|c|}{$\begin{array}{l}\text { For all } 97 \text { occurrences of no commu- } \\
\text { nication when transferring physician } \\
\text { expressed uncertainty (n, \%) }\end{array}$} & \multicolumn{2}{|c|}{$\begin{array}{l}\text { For all } 12 \text { occurrences of no commu- } \\
\text { nication when transferring physician } \\
\text { requested communication (n, \%) }\end{array}$} & \multicolumn{2}{|c|}{$\begin{array}{l}\text { For all } 119 \text { occurrences of no communica- } \\
\text { tion when clinical decisions changed (n, } \\
\%)\end{array}$} \\
\hline \multicolumn{2}{|c|}{ Case contextual factors } & \multicolumn{2}{|l|}{$38,39 \%$} & \multicolumn{2}{|l|}{$1,8 \%$} & \multicolumn{2}{|l|}{$38,32 \%$} \\
\hline \multicolumn{2}{|c|}{ Structural factors } & \multicolumn{2}{|l|}{$25,26 \%$} & \multicolumn{2}{|l|}{$8,67 \%$} & \multicolumn{2}{|l|}{$36,30 \%$} \\
\hline \multicolumn{2}{|c|}{ Interpersonal factors } & $14,14 \%$ & & \multicolumn{2}{|l|}{$0,0 \%$} & \multicolumn{2}{|l|}{$22,19 \%$} \\
\hline \multicolumn{2}{|c|}{ Other } & $20,21 \%$ & & \multicolumn{2}{|l|}{$3,25 \%$} & \multicolumn{2}{|l|}{$23,19 \%$} \\
\hline \multicolumn{2}{|l|}{ CATEGORY } & \multicolumn{6}{|c|}{ Examples of rationales for no communication across 3 conditions } \\
\hline \multicolumn{2}{|l|}{$\begin{array}{l}\text { Case contextual } \\
\text { factors }^{\mathrm{a}}\end{array}$} & \multicolumn{6}{|c|}{$\begin{array}{l}\text { - Mild shift in plan, course as expected } \\
\text { - Expected clinical evolution, anticipatory guidance was clear } \\
\text { - Nothing substantive, no major report to give back } \\
\text { - Low yield learning opportunity } \\
\text { - Clinically uninteresting } \\
\text { - Final diagnosis was in the realm of expected }\end{array}$} \\
\hline \multicolumn{2}{|c|}{ Structural factors ${ }^{b}$} & \multicolumn{6}{|c|}{$\begin{array}{l}\text { - Transferring physician on vacation, no opportunity to discuss } \\
\text { - Too soon, just made the change today } \\
\text { - Not enough information back to make the call } \\
\text { - Waiting for the final path report } \\
\text { - Too busy, diagnosis already on transferring physicians' differential } \\
\text { - Will sign patient back over to transferring physician }\end{array}$} \\
\hline \multicolumn{2}{|c|}{ Interpersonal factors ${ }^{\mathrm{C}}$} & \multicolumn{6}{|c|}{$\begin{array}{l}\text { - Expected transferring physician was chart stalking because he would be curious } \\
\text { - It would be uncomfortable [for me] to tell my boss he was wrong } \\
\text { - "Felt uneasy about our relationship" } \\
\text { - Didn't want transferring physician to feel judged about missing the issue } \\
\text { - Because of defensive posture, I only communicate major issues } \\
\text { - "She didn't ask, I didn't seek her out" }\end{array}$} \\
\hline \multicolumn{2}{|l|}{ Other ${ }^{d}$} & \multicolumn{6}{|c|}{$\begin{array}{l}\text { - Planned communication not yet occurred } \\
\text { - New work flow will [indirectly] come back to the group } \\
\text { - Not a priority to communicate } \\
\text { - Would potentially chat with transferring physician about this one } \\
\text { - Didn't think of it [communicating] } \\
\text { - It's how we operate [no communication culture] }\end{array}$} \\
\hline \multicolumn{8}{|c|}{$\begin{array}{l}\text { aRationales pertaining to the clinical case, including judgments about the learning value for the transferring physician } \\
\text { bRationales pertaining to structural barriers including clinical information not yet available, no opportunity to communicate (i.e. on vacation), time constraints, or } \\
\text { communication is scheduled in the future because the current physician will be transitioning patients back to the same physician } \\
\text { 'Rationales pertaining to interpersonal factors including barriers related to hierarchy, familiarity with how the transferring physician will react, or no established } \\
\text { relationship } \\
\text { dRationales that did not fit into the above categories including references to the culture of communication or hedging efforts to communicate. }\end{array}$} \\
\hline
\end{tabular}

nication culture also contributed to the low communication rate.

As we consider implications of communication back for transferring physicians' learning, a lack of communication may not always be problematic. When the current physician's clinical reasoning processes align with those of the transferring physician, uncertainty is not expressed, and nothing untoward happened to the patient, it may be that efforts to communicate back exceeded the perceived value of doing so. However, under conditions of transferring physicians' uncertainty at the time of care transitions or current physicians' changed clinical reasoning decisions after transitions, communicating back should be useful for improving care and learning [5].
Tracking patient outcomes is a learning habit of master clinicians [4, 31]. Experience of uncertainty from any source-evolution of the patient's illness, clinical ambiguity, incomplete information, or anticipated but unknown changes to clinical decisions-leads some transferring physicians to seek clinical feedback [8]. We found that transferring physicians commonly initiated communication with current physicians. Although our study was not designed to explore transferring physicians' reasons for initiating communication with current physicians, they may be motivated to reduce their uncertainty. Contrarily, it may be that physicians who transitioned off service simply engaged the current physician before the current physician had time to initiate the communication. Although not explicitly stated, a re- 
quest for communication back could be interpreted as the transferring physicians' desire for clinical feedback. In either case, transferring physicians may be signaling their receptivity to clinical feedback.

If transferring physicians seek follow-up on previous clinical decisions, either by chart review[9] or initiating communication, then what is the role of the current physician in providing clinical feedback? It would seem that the learning opportunities for transferring physicians most at risk of getting lost in discontinuous care settings are those where transferring physicians do not track patients' outcomes, anticipating low learning value [8]. In these situations, current physicians may be the only source of clinical feedback that could improve care and learning. Our findings suggest two specific conditions where transferring physicians' learning opportunities may have been lost because communication did not occur: when current physicians changed transferring physicians' clinical reasoning decisions or transferring physicians expressed uncertainty at the transition. Only one of these, perceptions of expressed uncertainty, was significantly associated with communication. From a situated cognition perspective, transferring physicians' expressions of uncertainty places responsibility on current physicians to provide clinical feedback to build shared knowledge and insights in ways that benefit patients.

What did we learn about conditions that influence communication and could be modified to improve learning in discontinuous care settings? Notably, case contextual factors were the driving force for most instances of both communication and non-communication. In some cases, current physicians made judgments about the informational value to transferring physicians. That clinical cases evolved and turned out "as expected" may suggest there is nothing left to learn. Physicians make such judgments based on their personal, situated interpretations of prior experiences, which likely differ between colleagues [7, 19]. Communication for the purpose of learning may correct erroneous or confirm effective reasoning processes. If left unaddressed, a lack of communication may unknowingly reinforce poor decisions and limit physicians' abilities to more accurately predict future events [32]. Although more research is needed to understand these issues, our results suggest that clinical feedback communication might improve the quality of care patients receive in discontinuous care settings and should be a work expectation.

Structures posed common barriers to communication in the three conditions of interest in this study. Time constraints suggest physicians made trade-offs when communication did not occur. When communication cannot occur conveniently, it may require effort physicians feel they cannot afford to invest. The intent to communicate may simply get lost in the shuffle of competing demands. Work schedules facilitated and inhibited communication. Physicians transitioning patients between them work opposite schedules and are often unavailable during their 'off' weeks. Contrarily, physicians working opposite weeks are sometimes scheduled to transition back-to-back, creating a scheduled communication opportunity. Future investigations could explore ways to overcome these structural barriers to communication.

\section{Limitations}

Our methodological choices place limits on how broadly we might speculate about our contribution. We asked participants to indicate if communication occurred with transferring physicians, suggesting our findings represented conscious communication decisions when perhaps they did not. We simultaneously collected data from current physicians about their communication decisions and their perceptions of the transferring physicians' uncertainty and request for communication. It is possible their communication behavior influenced answers to these interview questions. We purposefully scheduled our interviews to include a time interval between transition and interview to allow the current physician's clinical reasoning processes to take place. We relied on current physicians' perceptions of transferring physicians' uncertainty and request for communication. Future studies could explore both views of transition communication by 1) witnessing the actual communication at transitions, and 2) following up with both the transferring and current physicians.

\section{Conclusion}

In clinical learning environments where frequent transitions of responsibility interrupt transferring physicians' clinical reasoning processes, current physicians' communication back to transferring physicians about evolving clinical outcomes may be an important source of clinical feedback. Knowing the outcomes of previous reasoning may facilitate learning and improve patient care. Yet, current physicians in this study communicated infrequently, raising questions about lost learning opportunities. Some of the structural and interpersonal barriers to communication we identified may be amenable to changes. We suggest physicians start with creating expectations for clinical feedback communication and refining their care transition communication processes to include clarifying transferring physicians' uncertainty and desire for communication back, which may reduce some of the barriers to follow-up communication.

Acknowledgements The authors wish to thank the study participants for generously volunteering their time in support of this research.

Conflict of interest J.L. Bowen, J. Chiovaro, B.C. O'Brien, C.K. Boscardin, D.M. Irby andO. ten Cate declare that they have no competing interests. 
Open Access This article is licensed under a Creative Commons Attribution 4.0 International License, which permits use, sharing, adaptation, distribution and reproduction in any medium or format, as long as you give appropriate credit to the original author(s) and the source, provide a link to the Creative Commons licence, and indicate if changes were made. The images or other third party material in this article are included in the article's Creative Commons licence, unless indicated otherwise in a credit line to the material. If material is not included in the article's Creative Commons licence and your intended use is not permitted by statutory regulation or exceeds the permitted use, you will need to obtain permission directly from the copyright holder. To view a copy of this licence, visit http://creativecommons.org/licenses/by/4.0/.

\section{References}

1. Ludmerer KM. Time to Heal. New York: Oxford University Press; 1999.

2. Wachter RM, Goldman L. The emerging role of "hospitalists" in the American health care system. N Engl J Med. 1996;335:514-7.

3. Asch DA, Parker RM. The Libby Zion case. One step forward or two steps backward? NEnglJ Med. 1988;318:771-5.

4. Murthy VK, O'Brien B, Dhaliwal G. An inquiry into the early careers of master clinicians. J Grad Med Educ. 2018;10:500-6.

5. Schiff GD. Minimizing diagnostic error: the importance of follow-up and feedback. Am J Med. 2008;121(5 Suppl):S38-S42.

6. Narayana S, Rajkomar A, Harrison JD, Valencia V, Dhaliwal G, Ranji SR. What happened to my patient? An educational intervention to facilitate postdischarge patient follow-up. J Grad Med Educ. 2017;9:627-33.

7. Teunissen PW. Experience, trajectories, and reifications: an emerging framework of practice-based learning in healthcare workplaces. Adv Health Sci Educ Theory Pract. 2015;20:843-56.

8. Bowen JL, Ilgen JS, Irby DM, Ten Cate O, O’Brien BC. “You have to know the end of the story": Motivations to follow up after transitions of clinical responsibility. Acad Med. 2017;92(11S):S48-S54.

9. Bowen JL, O'Brien BC, Ilgen JS, Irby DM, Ten Cate O. Chart stalking, list making, and physicians' efforts to track patients' outcomes after transitioning responsibility. Med Educ. 2018;52:404-13.

10. Arora VM, Manjarrez E, Dressler DD, Basaviah P, Halasyamani L, Kripalani S. Hospitalist handoffs: a systematic review and task force recommendations. J Hosp Med. 2009;4:433-40.

11. Frankel RM, Flanagan M, Ebright P, et al. Context, culture and (non-verbal) communication affect handover quality. BMJ Qual Saf. 2012;21 (Suppl 1):i121-i8.

12. Young JQ, Wachter RM, Ten Cate O, O'Sullivan PS, Irby DM. Advancing the next generation of handover research and practice with cognitive load theory. BMJ Qual Saf. 2016;25:66-70.

13. Apker J, Mallak LA, Gibson SC. Communicating in the "gray zone": perceptions about emergency physician hospitalist handoffs and patient safety. Acad Emerg Med. 2007;14:884-94.

14. Arora V, Johnson J, Lovinger D, Humphrey HJ, Meltzer DO. Communication failures in patient sign-out and sugges- tions for improvement: a critical incident analysis. Qual Saf Health Care. 2005;14:401-7.

15. Hinami K, Farnan JM, Meltzer DO, Arora VM. Understanding communication during hospitalist service changes: a mixed methods study. JHosp Med. 2009;4:535-40.

16. Militello LG, RattrayNA, Flanagan ME, etal. "Workin' on our night moves": How residents prepare for shift handoffs. Jt CommJ Qual PatientSaf. 2018;44:485-93.

17. Vanka A, Farris G, Bortinger J, Huang G, Mattison ML. Mind the gap: a novel care transitions curriculum for hospitalists and residents. J Grad Med Educ. 2015;7:277-8.

18. Orlander JD, Fincke BG. Morbidity and mortality conference: a survey of academic internal medicine departments. J Gen Intern Med. 2003;18:656-8.

19. Brown JS, Collins A, Duguid P. Situated cognition and the culture oflearning. Educ Res. 1989;19:32-42.

20. Torre D, Durning SJ. Social cognitive theory: thinking and learning in social settings. In: Cleland J, Durning SJ, editors. Researching medical education. West Sussex: Wiley; 2015. pp. 105-16.

21. Bowen JL, Boscardin CK, Chiovaro J, et al. A view from the sender side of feedback: anticipated receptivity to clinical feedback when changing prior physicians' clinical decisions-a mixed methods study. Adv Health Sci Educ Theory Pract. 2019; https://doi.org/10.1007/s10459-01909916-2.

22. Ilgen JS, Eva KW, de Bruin A, Cook DA, Regehr G. Comfort with uncertainty: reframing our conceptions of how clinicians navigate complex clinical situations. Adv Health Sci Educ Theory Pract. 2019;24:797-809.

23. Bing-You RG, Paterson J, Levine MA. Feedback falling on deaf ears: residents' receptivity to feedback tempered by sender credibility. Med Teach. 1997;19:40-4.

24. Eva KW, Armson H, Holmboe E, et al. Factors influencing responsiveness to feedback: on the interplay between fear, confidence, and reasoning processes. Adv Health Sci Educ Theory Pract. 2012;17:15-26.

25. Bowen JL, Ilgen JS, Regehr G, Ten Cate O, Irby DM, O'Brien BC. Reflections from the rear view mirror: internal medicine physicians' reactions to clinical feedback after transitions of responsibility. Acad Med. 2019;94:1953-60.

26. Cook DA, Bordage G, Schmidt HG. Description, justification and clarification: a framework for classifying the purposes of research in medical education. Med Educ. 2008;42:128-33.

27. Creswell JW, Plano Clark VL. Designing and conducting mixed methods research. 3rd ed. Los Angeles: SAGE; 2018.

28. Paradis E, Varpio L. Difficult but important questions about the ethics of qualitative research. Perspect Med Educ. 2018;7:65-6.

29. Miles M, Huberman A, Saldana J. Qualitative data analysis: a methods sourcebook. Los Angeles: SAGE; 2014.

30. Monrouxe LV, Rees CE. When I say ... quantification in qualitative research. Med Educ. 2019; https://doi.org/10. $1111 /$ medu.14010.

31. Sargeant J, Mann K, Sinclair D, et al. Learning in practice: experiences and perceptions of high-scoring physicians. Acad Med. 2006;81:655-60.

32. Overoye AL, Storm BC. Harnessing the power of uncertainty to enhance learning. Transl Issues Psychol Sci. 2015;1:140-8. 